\title{
Scoliosis in Children: Impact of Goal-Directed Therapies on Intraoperative and Postoperative Outcomes
}

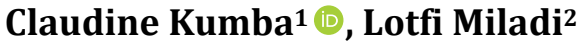 \\ ${ }^{1}$ Department of Pediatric and Obstetric Anesthesia and Critical Care, Hôpital Universitaire Necker Enfants Malades, Assistance \\ Publique Hôpitaux de Paris, APHP, Université de Paris, Paris, France \\ ${ }^{2}$ Department of Pediatric Orthopedic Surgery, Hôpital Universitaire Necker Enfants Malades, Assistance Publique Hôpitaux de \\ Paris, APHP, Université de Paris, Paris, France \\ Email: lotfi.miladi@aphp.fr, claudine.kumba@gmail.com, claudine.kumba@aphp.fr
}

How to cite this paper: Kumba, C. and Miladi, L. (2021) Scoliosis in Children: Impact of Goal-Directed Therapies on Intraoperative and Postoperative Outcomes. Open Journal of Orthopedics, 11, 315-326. https://doi.org/10.4236/ojo.2021.1110030

Received: September 24, 2021

Accepted: October 22, 2021

Published: October 25, 2021

Copyright $\odot 2021$ by author(s) and Scientific Research Publishing Inc. This work is licensed under the Creative Commons Attribution International License (CC BY 4.0).

http://creativecommons.org/licenses/by/4.0/ (c) (i) Open Access

\begin{abstract}
Background: Scoliosis is among interventions with high postoperative complication rates due to the characteristics of the surgery, where blood loss, transfusion and fluid requirements can be increased. A monocentric retrospective observational study was undertaken earlier to determine predictors of intraoperative and postoperative outcomes in surgical patients. In this initial cohort, there were patients who underwent scoliosis surgery, and a secondary analysis to describe outcomes in these patients was realized and presented here. Objective: To describe intraoperative and postoperative outcomes in patients under 18 years old in scoliosis surgery included in the initial study and to propose improvement and implementation measures. Methods: A secondary analysis of patients undergoing scoliosis surgery from 1 January 2014 to 17 May 2017 was undertaken in our institution-Necker Enfants Malades university hospital. The study was approved by the Ethics Committee. Results: There were 116 patients with a mean age of $147.5 \pm 40.2$ months. Twenty-eight patients (24.1\%) presented intraoperative and/or postoperative complications. The most common intraoperative complication was hemorrhagic shock in 3 patients (2.6\%). The most common postoperative organ failure was neurologic in seven patients $(6 \%)$, respiratory in 3 patients $(2.6 \%)$, cardio-circulatory in 2 patients $(1.7 \%)$ and renal failure in 1 patient $(0.9 \%)$. The most common postoperative infection was surgical wound sepsis in 8 patients (6.9\%), urinary sepsis in 3 patients (2.6\%), and abdominal sepsis and septicemia in 2 patients (1.7\%). 12 patients (10.3\%) had reoperations. Fifty-six patients (48.3\%) had intraoperative transfusion. There was no in-hospital mortality. Conclusion: The portion of patients with intraoperative and or postoperative complications was
\end{abstract}


$24.1 \%$, integrating goal-directed therapies in this surgical setting could improve postoperative outcomes.

\section{Keywords}

Scoliosis, Children, Outcome, Goal-Directed Therapies

\section{Introduction}

Scoliosis surgery is one of the most commonly performed major elective surgeries in our hospital.

This disorder of the vertebral column is classified as idiopathic or juvenile and non-idiopathic scoliosis (see Figure 1 and Figure 2 illustrating spine radiography in a 13-year-old child with neuromuscular scoliosis before and after a mini-invasive surgical technique).

The etiopathology of juvenile scoliosis is unknown, whereas non-idiopathic scoliosis is associated with neuromuscular diseases, bone diseases or other syndromes. Postoperative morbidity in scoliosis is high due to the characteristics of the surgery, where blood losses, transfusion and fluid therapy requirements can

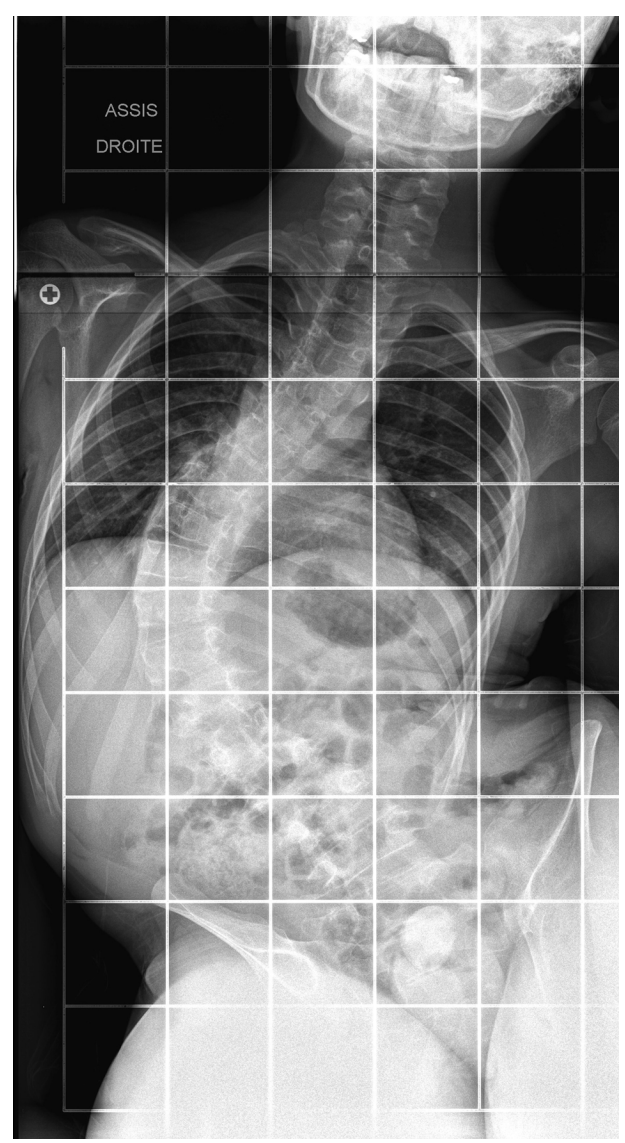

Figure 1. Preoperative spine radiography of a 13-year-old child with neuromuscular scoliosis. 


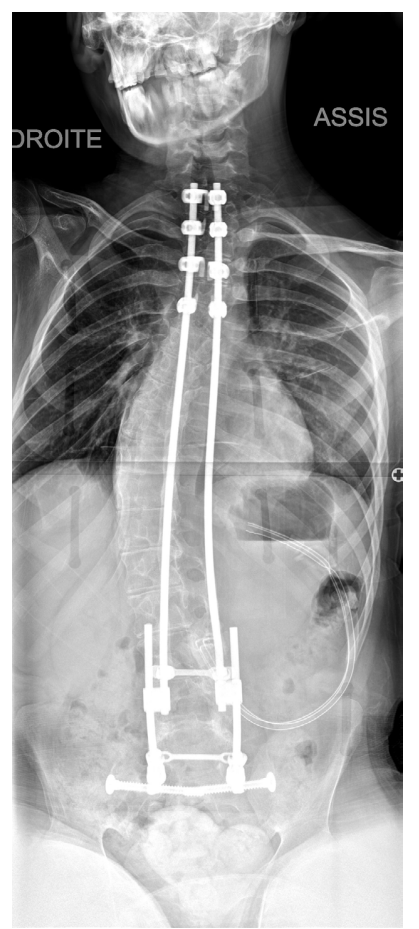

Figure 2. Postoperative spine radiography of a 13-year-old child with neuromuscular scoliosis after a minimally invasive surgical technique.

be increased [1]. The patient's general status is one of the predictors of postoperative evolution [2]. There is growing evidence that applying Enhanced Recovery after Surgery (ERAS) protocols in scoliosis surgery in children reduces postoperative morbidity in terms of organ dysfunction and Length of Hospital Stay (LOS) [3]. ERAS protocols integrate different patient management aspects that have the objectives to enhance recovery after surgery [3]. These include among other aspects, intraoperative transfusion protocols guided with the point of care viscoelastic methods in hemorrhagic settings, optimal intraoperative fluid therapy management using validated tools in children, intraoperative protective mechanical ventilation methods, minimally invasive surgical techniques and optimal intraoperative and postoperative pain management [3]-[9]. In our hospital, we conducted a monocentric observational study in neurosurgical, abdominal surgical and orthopedic patients to determine predictors of intraoperative and postoperative outcomes [2]. In this cohort of 594 patients, some patients underwent scoliosis surgery. We aimed with this secondary analysis of the initial study to describe intraoperative and postoperative outcomes in patients who underwent scoliosis surgery and to implement improved intraoperative patient management protocols with the objectives of optimizing postoperative outcomes in this surgical population.

\section{Materials and Methods}

A secondary analysis of patients who underwent scoliosis surgery was included in the initial study [2]. 
The study was registered to the National Commission for Computer Science and Liberties (CNIL) under the number 2028257 v0 on 21 February 2017 and approved by the Ethics Committee of our hospital under the registration number 2017-CK-5-R1 on 21 March 2017.

The inclusion criteria were patients included in the initial study aged less than 18 years old who underwent scoliosis surgery.

The exclusion criteria were patients above 18 years old who did not undergo scoliosis surgery and who were included in the initial study.

Patients were included retrospectively from 1 January 2014 to 17 May 2017.

In our hospital, scoliosis surgical patients were managed perioperatively according to a protocol described here. Preoperatively, patients had respiratory functional tests, iron and erythropoietin supplementation, complete dental examination, nasal antibiotic therapy with mupirocin and a special fiber-free diet several days prior to surgery, complete blood cell count and packed red blood cell units available. Intraoperatively, 2 large peripheral intravenous lines and an arterial catheter were inserted; patients were monitored with bispectral index, indwelling bladder catheter, nasogastric tubing, central core temperature probe, fluid warming system, warming blanket and somesthesic evoked potentials. Induction of anesthesia could be inhalational with sevoflurane or intravenous with propofol, remifentanil and ketamine. Maintenance of anesthesia was intravenous with propofol, remifentanil and ketamine. The airway was secured with orotracheal or nasotracheal intubation. Tranexamic acid was administered as an intravenous bolus of $30 \mathrm{mg} / \mathrm{kg}$ followed by an intravenous infusion of $10 \mathrm{mg} / \mathrm{kg} / \mathrm{h}$. A cell saver was available in cases of non-idiopathic scoliosis. Antibiotic prophylaxis was performed with cefazolin and/or vancomycin depending on the patient's microbiological status. Fluid therapy was performed with crystalloids as a $10-20$ $\mathrm{ml} / \mathrm{kg}$ bolus and colloids (Plasmion ${ }^{\oplus}$ ) as a $30 \mathrm{ml} / \mathrm{kg}$ bolus. Intraoperative fluid management had the objectives of maintaining a mean arterial pressure above 60 $\mathrm{mmHg}$.

Postoperative analgesia was realized with spinal analgesia with morphine at 5 $\mu \mathrm{g} / \mathrm{kg}$ (maximum $500 \mu \mathrm{g}$ ) administered intraoperatively, acetaminophen, ketoprofen, clonidine and patient-controlled analgesia with intravenous bolus morphine.

Patients were extubated in the operating room or in the recovery room.

Statistics were analyzed with XLSTAT 2020.4.1 software. Continuous variables were expressed as medians with ranges or means with standard deviations. Categorial variables were described in proportions.

\section{Results}

Table 1 illustrates the general characteristics.

There were 116 patients with a mean age of $147.5 \pm 40.2$ months and a median weight of 34 kilograms $(24-45)$. Sixty-three patients (54.3\%) were American Society of Anesthesiologists grade 3 (ASA 3), and 17 patients (14.7\%) were ASA 4. Seventy-one patients (61.2\%) had neuromuscular or syndromic scoliosis, and 
Table 1. General characteristics.

\begin{tabular}{|c|c|}
\hline Characteristic & $\mathrm{N}=116$ \\
\hline Mean age \pm standard deviation in months & $147.5 \pm 40.2$ \\
\hline Median weight (interquartile range) in kilograms & $34(24-45)$ \\
\hline ASA I n (\%) & $1(0.9)$ \\
\hline ASA II n (\%) & $35(30)$ \\
\hline ASA III n (\%) & $63(54.3)$ \\
\hline ASA IV n (\%) & $17(14.7)$ \\
\hline Idiopathic scoliosis n (\%) & $45(38.8)$ \\
\hline Non idiopathic scoliosis $\mathrm{n}(\%)$ & $71(61.2)$ \\
\hline Emergency surgery n (\%) & $5(4.3)$ \\
\hline Elective surgery n (\%) & $111(95.7)$ \\
\hline Re-operation n (\%) & $12(10.3)$ \\
\hline $\begin{array}{l}\text { Patients with intra-operative and or postoperative complications } \\
\text { (organ failure or sepsis) } n(\%)\end{array}$ & $28(24.1)$ \\
\hline Intra-operative anaphylaxis $\mathrm{n}(\%)$ & $1(0.9)$ \\
\hline Intra-operative respiratory failure $\mathrm{n}(\%)$ & $1(0.9)$ \\
\hline Intra-operative hemorrhagic shock n (\%) & $3(2.6)$ \\
\hline Postoperative renal failure $\mathrm{n}(\%)$ & $1(0.9)$ \\
\hline Postoperative cardio-circulatory failure $\mathrm{n}(\%)$ & $2(1.7)$ \\
\hline Postoperative respiratory failure n (\%) & $3(2.6)$ \\
\hline Postoperative neurologic failure $\mathrm{n}(\%)$ & $7(6)$ \\
\hline Postoperative abdominal sepsis n (\%) & $2(1.7)$ \\
\hline Postoperative septicemia n (\%) & $2(1.7)$ \\
\hline Postoperative urinary sepsis n (\%) & $3(2.6)$ \\
\hline Postoperative surgical wound sepsis n (\%) & $8(6.9)$ \\
\hline In-hospital mortality n (\%) & $0(0)$ \\
\hline Transfusion $\mathrm{n}(\%)$ & $56(48.3)$ \\
\hline Median length of intensive care unit stay in days (range) & $4(0-25)$ \\
\hline Median length of hospital stay in days (range) & $10(1-50)$ \\
\hline Median total length of hospital stay in days (range) & $12(2-67)$ \\
\hline Median length of mechanical ventilation in days (range) & $0(0-21)$ \\
\hline Median packed red blood cells volume in units (range) & $0(0-3)$ \\
\hline Median fresh frozen plasma volume in units (range) & $0(0-5)$ \\
\hline Median concentrated platelet units (range) & $0(0-2)$ \\
\hline Mean preoperative hemoglobin levels \pm standard deviation in $\mathrm{g} / \mathrm{dL}$ & $12.4 \pm 1.7$ \\
\hline Mean postoperative hemoglobin levels \pm standard deviation in $\mathrm{g} / \mathrm{dL}$ & $10.7 \pm 1.6$ \\
\hline Median crystalloid volume in $\mathrm{ml}$ (range) & $1750(500-4684)$ \\
\hline Median colloid volume in ml (range) & $2000(0-6952)$ \\
\hline
\end{tabular}


forty-five patients (38.8\%) had idiopathic scoliosis. 111 patients $(95.7 \%)$ had elective surgery.

Twenty-eight patients (24.1\%) presented intraoperative and/or postoperative complications.

The most common intraoperative complication was hemorrhagic shock in 3 patients $(2.6 \%)$, followed by anaphylaxis and respiratory failure in one patient $(0.9 \%)$. The most common postoperative organ failure was neurologic in seven patients $(6 \%)$, followed by respiratory in 3 patients $(2.6 \%)$, cardio-circulatory in 2 patients (1.7\%) and renal failure in one patient (0.9\%).

The most common postoperative infection was surgical wound sepsis in eight patients (6.9\%), followed by urinary sepsis in three patients (2.6\%), and followed by abdominal sepsis and septicemia in two patients (1.7\%).

Twelve patients (10.3\%) had reoperations.

Fifty-six patients (48.3\%) were intraoperatively transfused with packed red blood cells and/or fresh frozen plasma and/or platelet units.

There was no in-hospital mortality.

The median length of intensive care unit stay was $4(0-25)$ days. The median length of hospital stay was $10(1-50)$ days. The median total length of hospital stay was $12(2-67)$ days. The median length of mechanical ventilation was 0 (0 21) days.

Table 2 illustrates co-morbidities.

The most common comorbidities were cerebral anoxic lesions in 22 patients (18.9\%), followed by neurofibromatosis in 7 patients (6\%), myelomeningocele, osteogenesis imperfecta and polymalformative syndrome in 4 patients (3.5\%), and followed by congenital heart disease and psychomotor deficiency in 2 patients $(2.5 \%)$.

In this hemorrhagic surgery in our hospital, at the time of this study, we did not use transfusion protocols guided with point-of-care devices for blood product management and fluid therapy was guided using mean arterial blood pressure.

\section{Discussion}

The rate of patients with intraoperative and/or postoperative complications was high.

Including transfusion-guided protocols with point-of-care tests, goal-directed fluid and hemodynamic therapy with validated tools and parameters in children, integrating enhanced recovery after surgery protocols to optimize intraoperative management in scoliosis surgery could improve postoperative outcomes in this surgical setting [3]-[15].

To improve blood product administration practices in scoliosis surgery, transfusion-guided protocols with point-of-care viscoelastic assays should be included in this setting. Point-of-care tests in hemorrhagic interventions have been shown to reduce fresh frozen plasma administration and the length of hospital 
Table 2. Co-morbidities.

\begin{tabular}{|c|c|}
\hline Co-morbidity & Number of patients (\%) \\
\hline Central core myopathy & $1(0.9)$ \\
\hline Cerebral anoxic lesions & $22(18.9)$ \\
\hline Congenital heart disease & $3(2.6)$ \\
\hline Convulsive encephalopathy & $2(1.7)$ \\
\hline Di George syndrome & $2(1.7)$ \\
\hline Ewing's sarcoma & $1(0.9)$ \\
\hline Goldenhar's syndrome & $1(0.9)$ \\
\hline Gorlin's syndrome & $2(1.7)$ \\
\hline Hurler's syndrome & $2(1.7)$ \\
\hline Muscular dystrophy & $2(1.7)$ \\
\hline Myelomeningocele & $4(3.5)$ \\
\hline Neurofibromatosis & $7(6.0)$ \\
\hline Osteogenesis imperfecta & $4(3.5)$ \\
\hline Pierre Robin syndrome & $2(1.7)$ \\
\hline Polymalformative syndrome & $4(3.5)$ \\
\hline Polytrauma & $2(1.7)$ \\
\hline Prader-Willi syndrome & $2(1.7)$ \\
\hline Psychomotor deficiency & $3(2.5)$ \\
\hline Scoliosis & $1(0.9)$ \\
\hline Sepsis & $1(0.9)$ \\
\hline Spina bifida & $2(1.7)$ \\
\hline Spinal muscular amyotrophy & $2(1.7)$ \\
\hline West syndrome & $1(0.9)$ \\
\hline Williams syndrome & $1(0.9)$ \\
\hline Xeroderma pigmentosum & $1(0.9)$ \\
\hline
\end{tabular}

stay in children [4]. Algorithms with rotational thromboelastometry are illustrated in Figures 3-5 to guide transfusion in hemorrhagic surgery in children under 2 years, in children between 2 to 16 years and in children over 16 years [15].

Intraoperative goal-directed fluid and hemodynamic therapy protocols with validated tools and parameters in children need to be included in intraoperative management in this surgical intervention to guide fluid and vasoactive therapy. Monitoring mean blood pressure is not enough for optimal hemodynamic management. Fluid management is best assessed with aortic blood flow peak velocity variation with echocardiography or esophageal Doppler probes [13]. The limiting factor of echocardiography is expertise. Transthoracic echocardiography can be difficult to perform if the patient is in the prone position, which can limit 


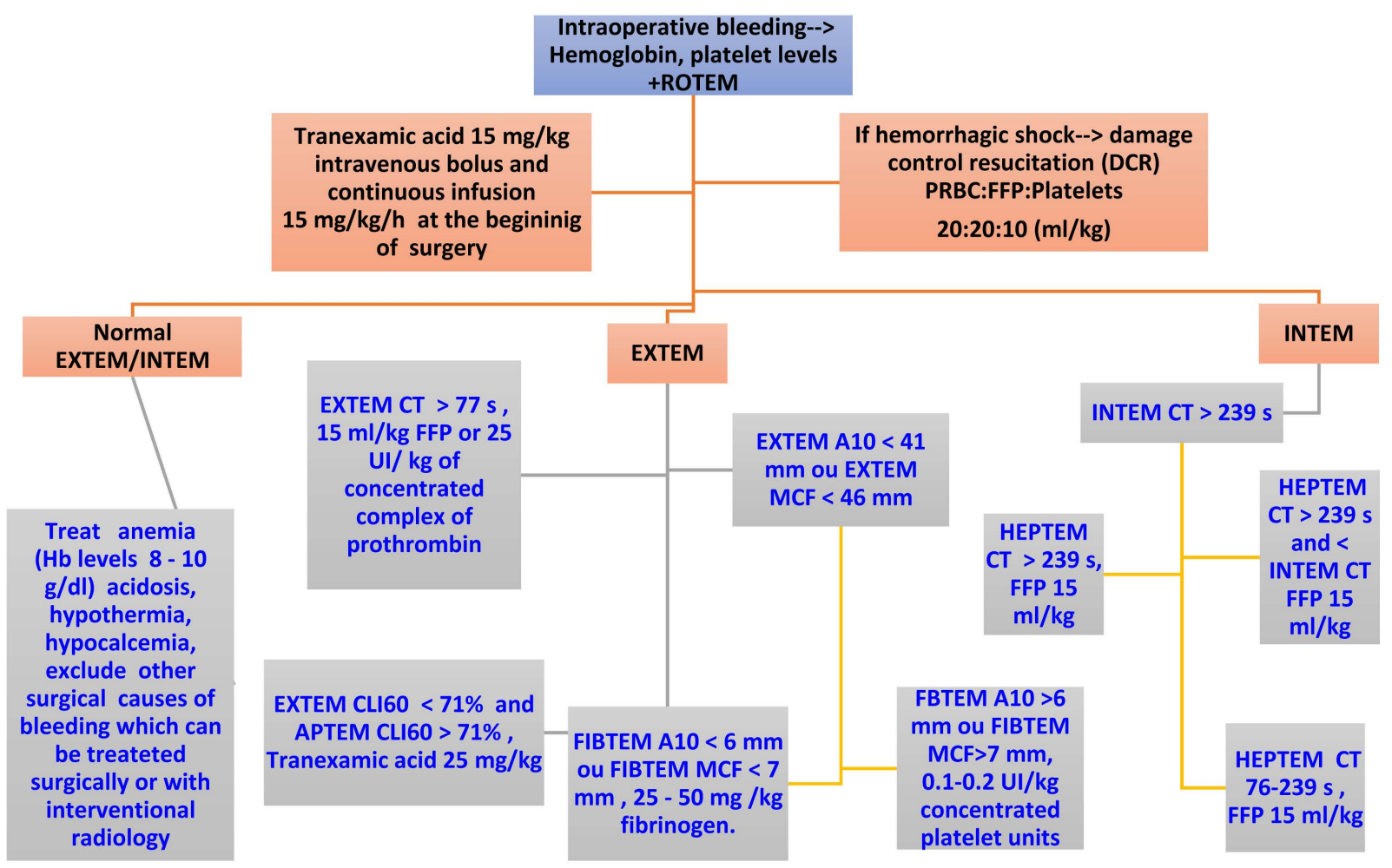

Figure 3. ROTEM algorithm in children between $0-24$ months. CT = coagulation time in seconds; A $10=$ clot firmness at 10 minutes; $\mathrm{MCF}=$ maximum clot firmness; CLI $60=$ lysis index in $\% 60$ minutes after CT; $\mathrm{ML}=$ maximum lysis in $\%$; FFP = fresh frozen plasma; $\mathrm{PRBC}=$ packed red blood cells; $\mathrm{Hb}=$ hemoglobin.

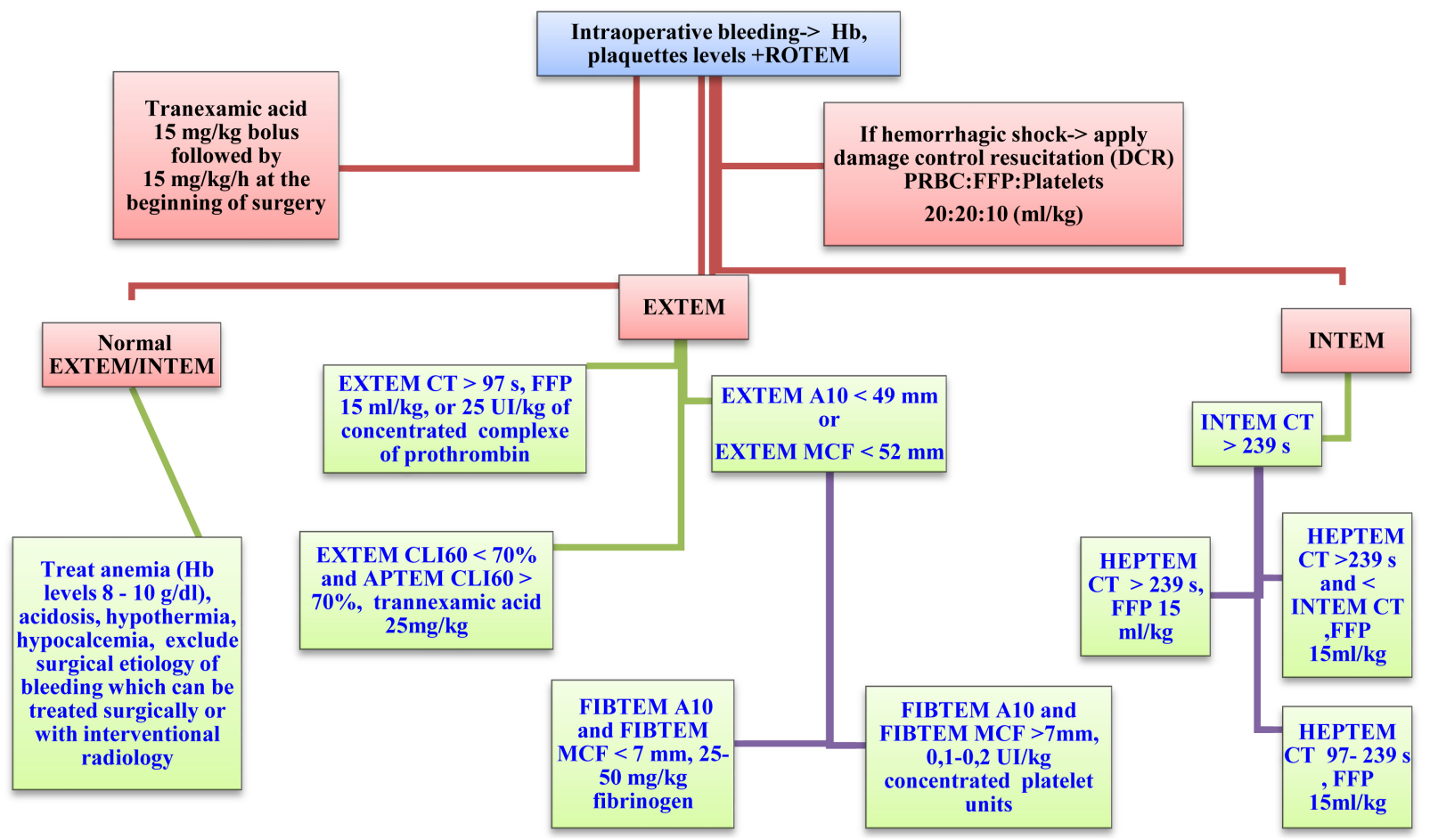

Figure 4. ROTEM algorithm in children between $2-16$ years. CT $=$ coagulation time in seconds; A $10=$ clot firmness after 10 minutes; $\mathrm{MCF}=$ maximum clot firmness; CLI60 = lysis index in \% 60 minutes after CT; ML = maximum lysis in \%; FFP = fresh frozen plasma; $\mathrm{PRBC}=$ packed red blood cells; $\mathrm{Hb}=$ hemoglobin. 


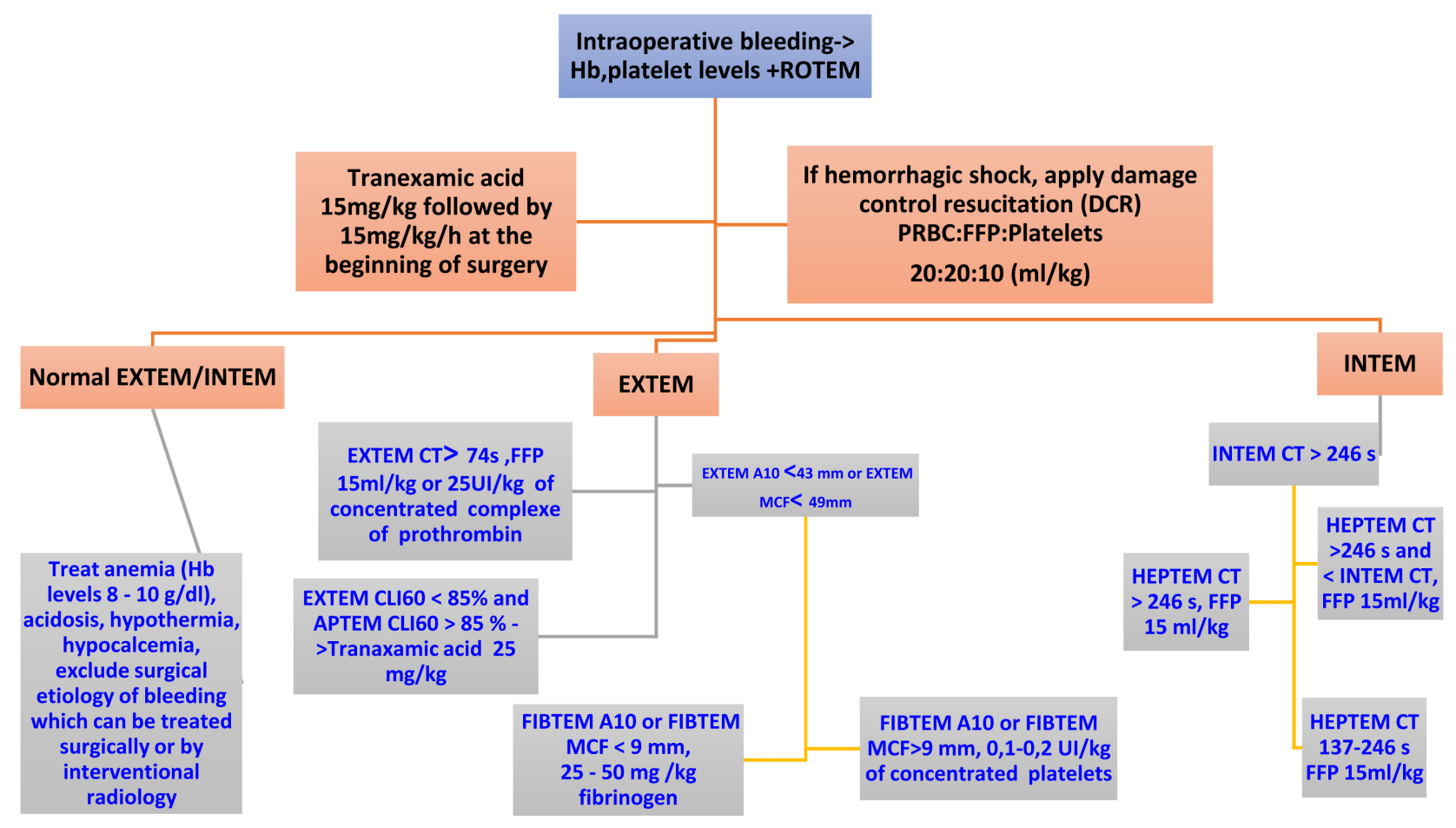

Figure 5. ROTEM algorithm $>16$ years. $\mathrm{CT}=$ coagulation time in seconds; $\mathrm{A} 10=$ clot firmness at 10 minutes; $\mathrm{MCF}=$ maximum clot firmness; CLI60 = lysis index in \% 60 minutes after CT; ML = maximum lysis in \%; FFP = fresh frozen plasma; PRBC = packed red blood cells; $\mathrm{Hb}=$ hemoglobin.

access to the apex or the sternal notch, and the solution can be either an esophageal Doppler probe or transesophageal echocardiography. However, the latter necessitates expertise. Flotrac/Vigileo has been shown to reduce blood product administration, fluid requirements, postoperative pulmonary and gastrointestinal complications and length of intensive care unit stay in spine surgery [11]. Stroke volume variation with Flotrac/Vigileo can be used for fluid therapy. With this device, stroke volume variation is determined with arterial pressure waveform analysis; however, evidence in children with Flotrac/Vigileo on the impact of postoperative outcome is lacking [10] [12]. Studies in children with Flotrac/Vigileo are needed to clarify the impact on postoperative outcome.

Predictors of postoperative outcomes include the patient's global status, type of surgery, emergency age and transfusion [14]. Goal-directed therapies are possible solutions for postoperative outcome improvement in critically ill children and in major pediatric surgery.

Enhanced recovery after surgery protocols have been shown to decrease postoperative complications in scoliosis surgery in children, and integrating these pathways in this surgical setting can improve postoperative evolution [3]. Minimally invasive surgical techniques are part of ERAS protocols. Minimally invasive surgical techniques such as fusionless bipolar fixation of the spine with lumbar or pelvic distal anchoring according to the etiology of scoliosis could also improve postoperative outcomes in this major surgery (Figure 1 and Figure 2) [16]. 
The limit of our study is the retrospective aspect and the strength of this study is the sample size and a homogenous surgical cohort. The hypothesis of the impact of goal-directed therapies on postoperative outcomes needs to be confirmed by prospective studies where these protocols are integrated in the global intraoperative patient's management.

\section{Conclusion}

To improve postoperative outcomes in pediatric scoliosis surgery, transfusionguided protocols with point-of-care tests, goal-directed fluid and hemodynamic therapy with validated tools and parameters in children and enhanced recovery after surgery need to be included in this surgical setting management. Minimally invasive surgical techniques which are part of ERAS protocols, are also promising alternatives to reduce postoperative morbidity in this surgery. Prospective studies where goal-directed-therapies are integrated into intraoperative patient management need to be developed in children to clarify their impact on postoperative outcomes.

\section{Conflicts of Interest}

The authors declare no conflicts of interest.

\section{Presentation of Preliminary Results}

This manuscript has been registered as a preprint under the DOI registration number https://doi.org/10.21203/rs.3.rs-765785/v2 on Research Square, a preprint platform.

\section{Authors Contribution}

Claudine Kumba conceptualized and designed the study and drafted the initial manuscript. She designed the data collection instruments, collected data, carried out initial and final analyses.

Lotfi Miladi reviewed the manuscript.

\section{Ethics Approval}

This study received approval from the Ethics Committee of Necker on 21 March 2017 under registration number 2017-CK-5-R1 and waived patient consent.

\section{References}

[1] Kumba, C. (2019) A Retrospective Descriptive Cohort Study of Preoperative, Intraoperative and Postoperative Management of Children in Scoliosis Surgery. EC Anaesthesia, 5, 20-29.

[2] Kumba, C., Cresci, F., Picard, C., et al. (2017) Transfusion and Morbi-Mortality Factors: An Observational Descriptive Retrospective Pediatric Cohort Study. Journal of Anesthesia \& Critical Care: Open Access, 8, Article No. 00315. https://doi.org/10.15406/jaccoa.2017.08.00315 
[3] Kumba, C., et al. (2019) Rapid Recovery Pathways after Surgery in Children: A Systematic Review and Meta-Analysis. Medical Journal of Clinical Trials \& Case Studies, 3, Article ID: 000211.

[4] Kumba, C., Querciagrossa, S., Harte, C., Willems, A., et al. (2019) A Systematic Review and Meta-Analysis of Goal Directed Intra-Operative Transfusion Protocols Guided by Viscoelastic Methods and Perioperative Outcomes in Children. International Journal of Recent Scientific Research, 10, 31466-31471.

[5] Kumba, C., Willems, A., Querciagrossa, S., et al. (2019) A Systematic Review and Meta-Analysis of Intraoperative Goal Directed Fluid and Haemodynamic Therapy in Children and Postoperative Outcome. Journal of Emergency Medicine \& Critical Care, 5, 1-9. https://doi.org/10.13188/2469-4045.1000020

[6] Kumba, C. (2020) Physiology Principles Underlying Goal Directed Therapies in Children. Research in Pediatrics \& Neonatology, 4, 294-298. https://doi.org/10.31031/RPN.2020.04.000591

[7] Kumba, C. (2020) Rationale of Goal Directed Therapies in Children. Advances in Pediatric Research, 7, 42. https://doi.org/10.35248/2385-4529.20.7.35

[8] Kumba, C. (2019) Do Goal Directed Therapies Improve Postoperative Outcome in Children? (Perioperative Goal Directed Fluid and Hemodynamic Therapy; Transfusion Goal Directed Therapy Using Viscoelastic Methods and Enhanced Recovery after Surgery and Postoperative Outcome): A Study Research Protocol. Acta Scientific Paediatrics, 2, 17-19. https://doi.org/10.31080/ASPE.2019.02.0094

[9] Kumba, C. (2020) Goal Directed Fluid and Hemodynamic Therapy and Postoperative Outcomes in Children: Value of Transthoracic Echocardiographic Aortic Blood Flow Peak Velocity Variation: A Multi-Centre Randomized Controlled Trial Protocol. Advances in Pediatric Research, 7, 35. https://doi.org/10.35248/2385-4529.20.7.35

[10] Slagt, C., Malagon, I. and Groeneveld, A.B.J. (2014) Systematic Review of Uncalibrated Arterial Pressure Waveform Analysis to Determine Cardiac Output and Stroke Volume Variation. British Journal of Anaesthesia, 112, 626-637.

https://doi.org/10.1093/bja/aet429

[11] Bacchin, M.R., Ceria, C.M., Giannone, S., et al. (2016) Goal-Directed Fluid Therapy Based on Stroke Volume Variation in Patients Undergoing Major Spine Surgery in Prone Position. Spine, 41, E1131-E1137. https://doi.org/10.1097/BRS.0000000000001601

[12] Biais, M., Nouette-Gaulain, K., Roullet, S., et al. (2009) A Comparison of Stroke Volume Variation Measured by Vigileo/FloTrac System and Aortic Doppler Echocardiography. Anesthesia \& Analgesia, 109, 466-469.

https://doi.org/10.1213/ane.0b013e3181ac6dac

[13] Gan, H., Cannesson, M., Chandler, J.R. and Ansermino, J.M. (2013) Predicting Fluid Responsiveness in Children: A Systematic Review. Anesthesia \& Analgesia, 117, 1380-1392. https://doi.org/10.1213/ANE.0b013e3182a9557e

[14] Kumba, C., Lenoire, A., Cairet, P., Dogaru-Dedieu, E., Belloni, I. and Orliaguet, G. (2018) Is Transfusion an Independent Risk Factor of Postoperative Outcome in Pediatric Orthopedic Surgical Patients? A Retrospective Study. Journal of Emergency Medicine \& Critical Care, 4, 7. https://doi.org/10.13188/2469-4045.1000018

[15] Kumba, C. (2021) Liver Transplantation in Children and Impact of Intraoperative Goal Directed Therapies on Postoperative Outcome. Research Square.

https://doi.org/10.21203/rs.3.rs-744584/v1 
[16] Miladi, L., Gaume, M., Khouri, N., Johnson, M., Topouchian, V. and Glorion, C. (2018) Minimally Invasive Surgery for Neuromuscular Scoliosis. Spine, 43, E968-E975. https://doi.org/10.1097/BRS.0000000000002588 